
Paper 252
CCivil-Comp Press, 2010
Proceedings of the Tenth International Conference
on Computational Structures Technology,
B.H.V. Topping, J.M. Adam, F.J. Pallarés,
R. Bru and M.L. Romero, (Editors),
Civil-Comp Press, Stirlingshire, Scotland

\title{
Comparison of Different Finite Element Models for the Simulation of the Ring-Ball on Ring Test
}

\author{
J. Barredo ${ }^{1}$, L. Hermanns ${ }^{2}$, I. del Rey ${ }^{2}$, A. Fraile ${ }^{2}$ and E. Alarcón ${ }^{2}$ \\ ${ }^{1}$ Centre for Modelling in Mechanical Engineering (CEMIM-F2I2) \\ ${ }^{2}$ Department of Structural Mechanics and Industrial Constructions \\ Polytechnical University of Madrid, Spain
}

\begin{abstract}
Monocrystalline silicon wafers are widely used in photovoltaic industry. The trend towards thinner wafers leads to higher breakage rates in the production process. In this context, the characterization of the mechanical strength becomes necessary. Different fracture tests as the ring/ball on ring are carried out. This paper presents different ways to simulate these tests. Analytical methods are applied in a first step. Simplified FE models (with shell elements and an axisymmetric model) that take into account non linearities existing in the test are presented and a $3 \mathrm{~d}$ solid model is detailed. Results in terms of calculation time, stress distribution, adjustment to the tests and fitting to a Weibull distribution are compared.
\end{abstract}

Keywords: ball on ring test, monocrystalline silicon wafers, finite element model, shell elements, axisymmetry, solid elements, Weibull distribution, contact, anisotropy, large displacements.

\section{Introduction}

The use of efficient and clean energies is one of the priority objectives of current society. Solar energy arises as a reliable alternative since the sun is a free and almost unlimited source of energy. But the high costs of the solar module make a major implementation of this kind of energy more difficult. It's estimated that almost the $50 \%$ of the final cost of the module comes from the silicon wafer [1], [2]. An obvious way to reduce the cost is cutting thinner wafers. Therefore, current trend in photovoltaic industry is the use of thinner wafers with larger areas so the number of wafers broken in the cell production process has increased. Strength characterization becomes necessary in order to optimize the steps in the production process.

Many studies concerning the mechanical properties of silicon wafers have been carried out last years ([4], [6], [9], [11]). These studies are usually carried out by means of tests and the development of numerical models simulating them [9]. In 
previous studies, the authors employed the four line bending test ([4], [6]). Another type of test has been used in recent studies. In this test the wafer is supported by a ring and the load is applied with a ring of smaller diameter or a ball. Therefore, it's called the ring on ring test or the ball on ring test.

The simulation of the test has been carried out in different ways. Analytical methods taken from the literature have been applied. They are not able to reflect the particular characteristics of the test and the material [10]. It's necessary to take into account the anisotropic behaviour of monocrystalline silicon wafers and the non-linearities of the test due to large displacements and contact between the wafer and the rings. Thus, the finite element method has been used for the simulation.

On one side, a 3-d model using shell elements has been developed. This model considers the anisotropic behaviour but its capability to adequately describe the stress distribution across the thickness is very limited. On the other side, an axisymmetric FE model has also been developed. It doesn't take into account the anisotropic behaviour but it's possible to analyze the stress state through the thickness. Finally, a model using solid elements has been developed in order to consider all these issues. The main disadvantage is the high calculation time.

The paper shows the material and the test characteristics and a comparison of the ways to analyze test results.

\section{Mechanical properties of monocrystalline silicon wafers}

In order to get a better understanding of the whole study, a brief description of the monocrystalline silicon wafers properties and the way to obtain them is going to be explained.

Monocrystalline wafers come from a silicon ingot grown through the Czochralski process (CZ) employing pieces of purified silicon (polycrystalline silicon) as feedstock. The growth process starts when polycrystalline silicon pieces are melted in a crucible and a seed crystal is introduced into the melt. Then, the seed is spun while is moved vertically, removing form the melt. In this process, the liquid crystallises at the seed following the crystal directions of the original seed. Figure 1 shows two steps of the silicon ingot growth through the $\mathrm{Cz}$ process.
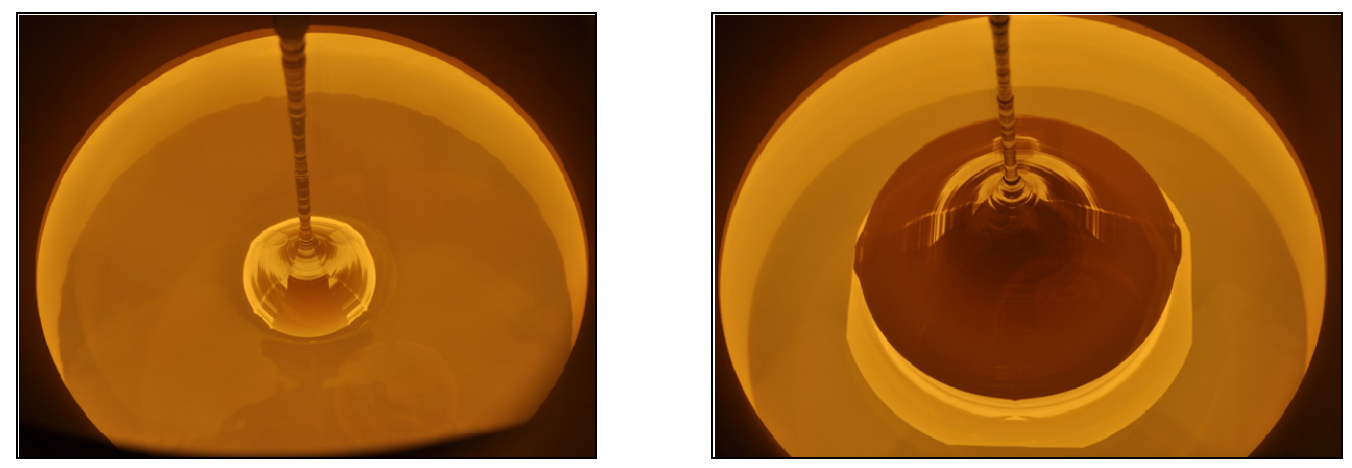

Figure 1: Czochralski growth process 
The final result of the process is a monocrystalline silicon ingot having a round cross section (figure 2).

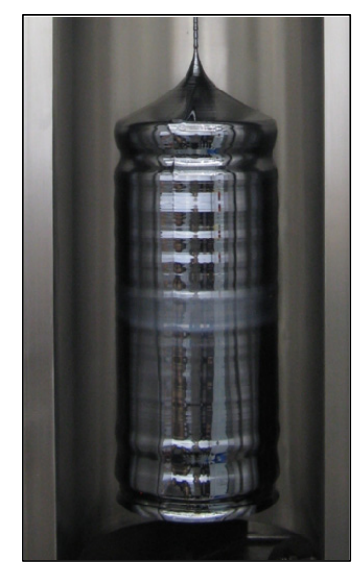

Figure 2. Silicon ingot growth through the $\mathrm{Cz}$ process

The ingot is pulled in a defined $<100>$ orientation. This is a big economic advantage since the solar cell process can use this crystallographic property to homogeneously texture solar cells with a very cost effective wet chemical etching step. By anisotropic etching a surface structure with random pyramids is built that couples the incoming light very effectively into the solar cell.

Square cells are best suited to build a highly efficient solar module whereas $\mathrm{Cz}$ ingots have a round cross section. In order to use both the crystal and the module area best, the ingots are usually cut into pseudo-square cross section by means of a saw. This step generates cracks on plane surface of the ingot.

Finally, the ingot is cut into several thousands of wafers in a process called wafering. The cutting process is carried out through a diamond wire bathed in slurry that contains silicon carbide particles. Again, this cutting process of the ingot generates cracks on the wafer surfaces.

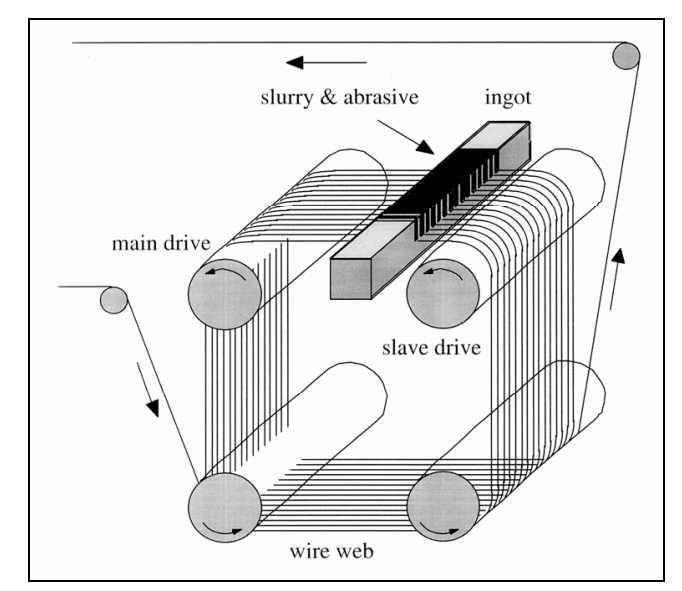

Figure 3. Multi wire sawing process. 
As can be seen, in the wafer generation process there are two steps that may reduce the mechanical strength of the wafer. The cutting process to get pseudo square cross section may cause cracks on surface of the ingot which at the end of the process will be wafers edges. Moreover, the wire cutting process may origin cracks on the wafer's surfaces. Therefore, the mechanical strength of silicon wafers depends on the cracks population residing in them ([4], [5], [6]).

\section{Tests}

The mechanical characterization of every material requires carrying out fracture tests. When choosing a test it is necessary to know the special features of the material. In this case, the tests have to take into account the two different cracks present in the wafer. To this end, two tests have been employed in order to characterize the mechanical strength.

\subsection{Four line bending test}

In the four line bending test, the wafer is placed on two supports and the load is applied through another two supports on the other side of the wafer. The supports can have cylindrical or blade shape and are equally separated of the central axis of the wafer. The test is schematized in figure 4.
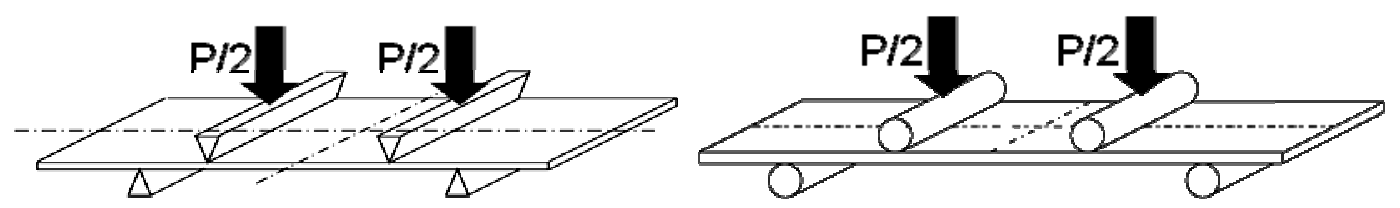

Figure 4. The four line bending test.

This test loads the zone between the lower supports so both types of cracks are stressed. Therefore, results are taking into account cracks in the central zone of the wafer generated by the wire sawing process as well as cracks near the edges generated by the cutting process to make pseudo square cross section wafers ([6], [9]).

This type of test has been employed by the authors in previous studies ([4], [6]) but it's not concerning to the results that are presented in this paper.

\subsection{Ball/ring on ring test}

In this test, wafer is supported on a ring and the load is applied on the other side of the wafer by means of a ring of smaller diameter or a ball. The test is schematized in figure 5 . 

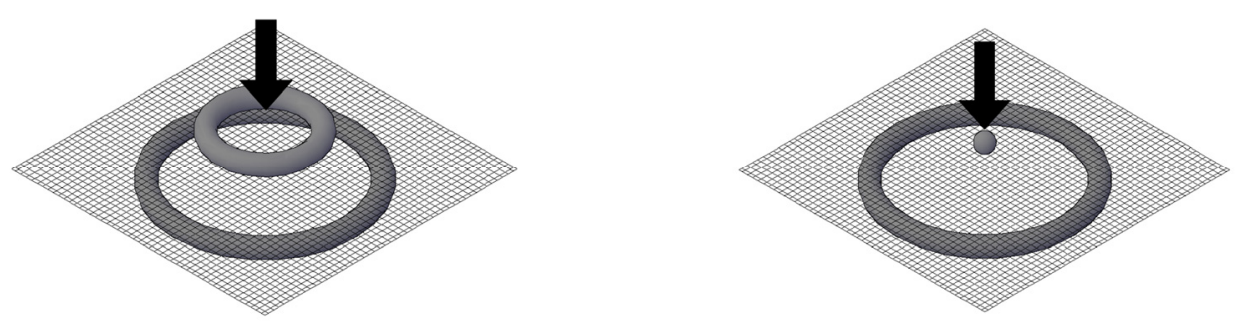

Figure 5. The ring on ring test and the ball on ring test

Stresses inside the lower ring are much higher than in the outer part so the failure is due to cracks located far away of the edges. Therefore, the evaluation of the strength using this type of test takes into account only cracks generated in the wafering process ([7], [9]).

This paper is related to the comparison of different numerical models developed to simulate this type of test so a more detailed description will be presented. In figure 6 photographs of both ring on ring and ball on ring test are shown.
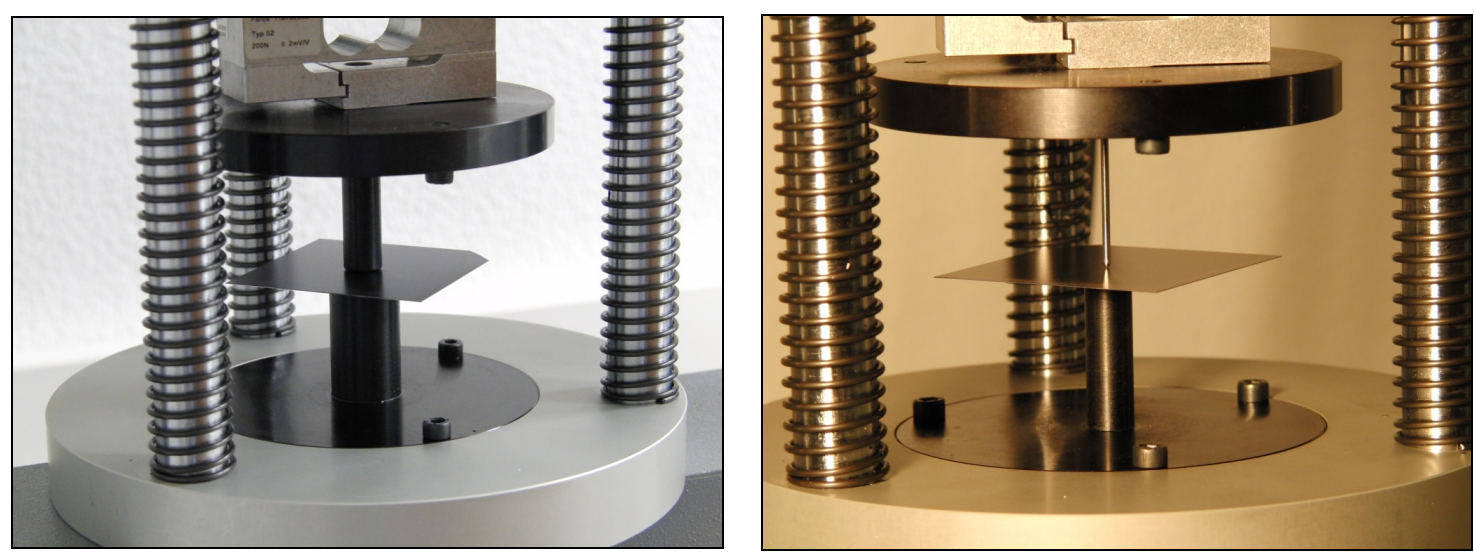

Figure 6. Photographs of the ring on ring and ball on ring test

In order to get a wide applicability for different sample dimensions, the testing machine has several rings of different diameters. The force transducer has a capability up to $200 \mathrm{~N}$. The displacement of the ball or the top ring is imposed and both the force and the displacement are recorded. In order to get a quasi-static test, the velocity is very low. In this study, it had a value of $5 \mu \mathrm{m} / \mathrm{s}$.

Wafers prepared for testing had an original thickness of approximately $250 \mu \mathrm{m}$. They were cut in four pieces by means of a laser giving four samples for each wafer of $62.5 \mathrm{~mm} \times 62.5 \mathrm{~mm}$ dimensions. Samples employed for this study were bathed in caustic soda during 6 minutes decreasing their thickness approximately $20 \mu \mathrm{m}$ per face. These samples were tested using the ball on ring test device. Test results are shown in figure 7. 


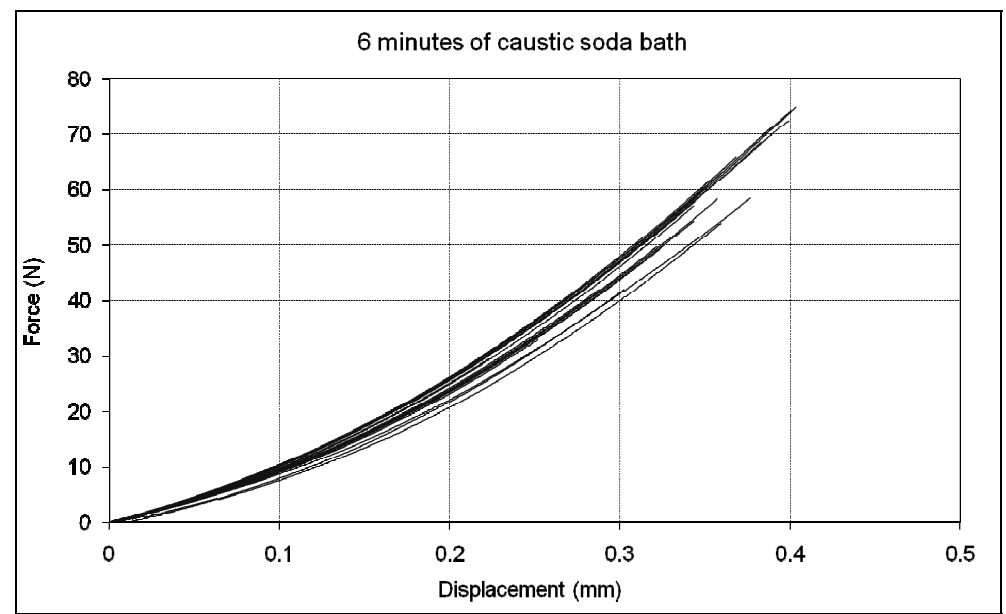

Figure 7. Results of the tests

The non-linear behaviour of the samples during the test can be clearly seen in figure 7. The different ways to obtain the maximum stress in the wafer at the failure moment are going to be presented ahead.

\section{$4 \quad$ Numerical models}

The test gives information about the behaviour of the samples and the maximum load and displacement that it reaches before the failure. It's necessary to know the stress that causes the failure. In this paper different ways to obtain the stress state of the wafer at the moment of failure are presented.

\subsection{Material properties}

First of all, it's necessary to know the material properties and the characteristics of the test and the samples.

The test chosen for the comparison is the ball on ring test with the following dimensions:

Lower ring diameter: $10 \mathrm{~mm}$

Diameter of the round cross section of the ring: $2 \mathrm{~mm}$

Diameter of the ball: $2 \mathrm{~mm}$

The sample is considered squared one in spite of the round corner that all samples have. This hypothesis is valid due to the very low values of stresses in the edge zones of the wafer. The dimensions of the sample are:

Geometry: $62.5 \mathrm{~mm}$ x $62.5 \mathrm{~mm}$

Thickness: $210 \mu \mathrm{m}$ (approximately) 
The monocrystalline silicon presents an anisotropic behaviour. The constants to represent it are:

$$
\begin{aligned}
& \mathrm{c} 11=165.6 \mathrm{GPa} \\
& \mathrm{c} 12=63.9 \mathrm{GPa} \\
& \mathrm{c} 44=79.5 \mathrm{GPa}
\end{aligned}
$$

This is all the information necessary to calculate the stress distribution in wafers during the test.

\subsection{Analytical method}

Some authors suggest the use of analytical methods to determine the maximum stress in the wafer. As a preliminary step, it may be useful because it's quick and easy. However, this analytical study is not valid for a good knowledge of the stress state of the wafer during the test. The anisotropy of the wafer is not taken into account and the non linear behaviour cannot be represented.

The maximum stress for the ball on ring test can be calculated with the following expression ([8], [10], [12]):

$$
\sigma_{\mathrm{r}}=\frac{3 \mathrm{~F}(1+v)}{4 \pi \mathrm{h}^{2}}\left[1+2 \operatorname{Ln}\left(\mathrm{r}_{2} / \mathrm{r}_{1}\right)+\frac{(1-v)}{(1+v)}\left(1-\frac{\mathrm{r}_{1}^{2}}{2 \mathrm{r}_{2}{ }^{2}}\right) \frac{\mathrm{r}_{2}^{2}}{\mathrm{r}_{\mathrm{eq}}{ }^{2}}\right]
$$

Where $\mathrm{F}$ is the fracture load; $\mathrm{h}$ is the thickness; $v$ is the Poisson coefficient; $r_{2}$ is the radius of the outer ring and $r_{e q}$ is the equivalent sample radius. For square samples the equivalent radius is:

$$
\mathrm{r}_{\mathrm{eq}}=\frac{\mathrm{L} / 2(1+\sqrt{2})}{2}=1.207 \mathrm{~L} / 2
$$

Where $\mathrm{L}$ is the side length of the sample; $\mathrm{r}_{1}$ corresponds to the radius of the contact zone between the ball and the sample. There are several expressions [10] to calculate it but since FE models have been developed in this study, the radius of the contact zone has been taken from the $3 \mathrm{~d}$ solid FE model. It's considered constant and has a value of $150 \mu \mathrm{m}$.

Through this expression, the maximum stress of each test may be quickly calculated. As already has been mentioned, it's necessary to take into account the anisotropic behaviour of mono-crystalline silicon wafers and the non-linearities of the test due to large displacements and contact between the wafer and the rings. Thus, the finite element method has been used for the simulation using the commercial package ANSYS. 


\subsection{Model 1: 3D with shell elements}

The first model presented is a 3D Finite Element Model in which wafer and supports have been modeled with four node shell elements. The anisotropy has been considered and the large displacements formulation has been employed. Additionally, contact between the sample and the ring and ball has been taken into account. The coefficient of friction used has a value of 0.35 . Contact algorithms require a fine mesh in the contact zone to avoid convergence problems. In order to reduce the number of elements, the mesh size is different depending on the zone of the wafer (figure 8).
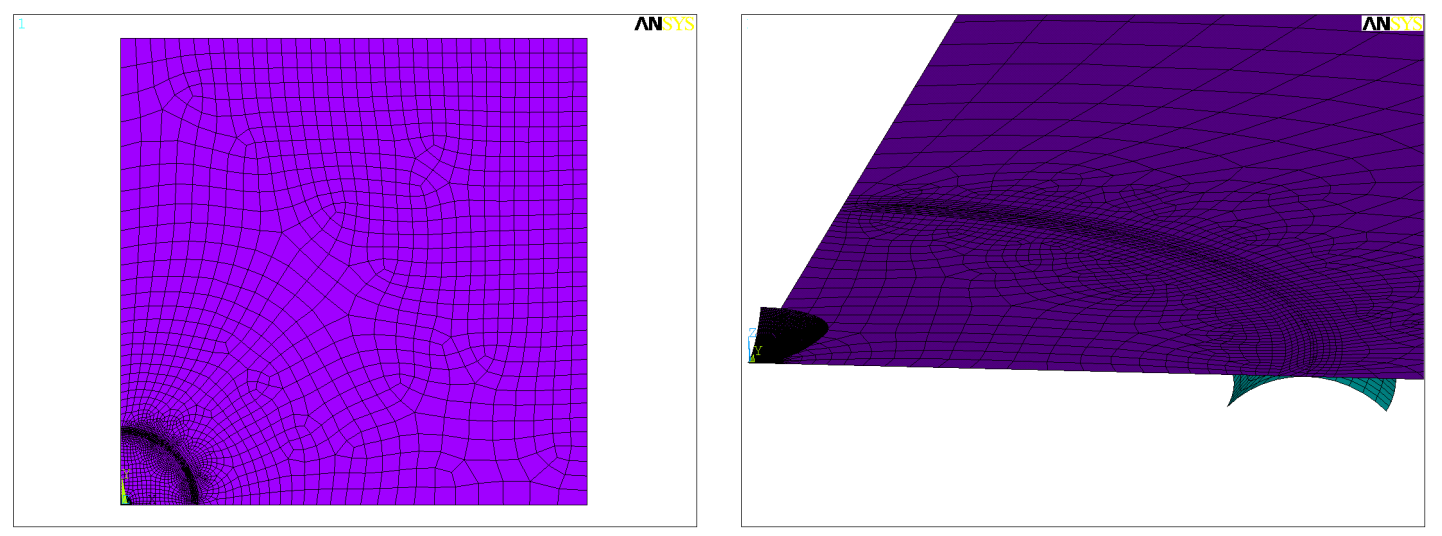

Figure 8. Mesh of the model 1

The model has 20239 elements and 11530 nodes. The calculation time has been 440 seconds.

Figure 9 shows the displacements of the wafer in the model. Non-concentric displacements are the consequence of the material anisotropy.

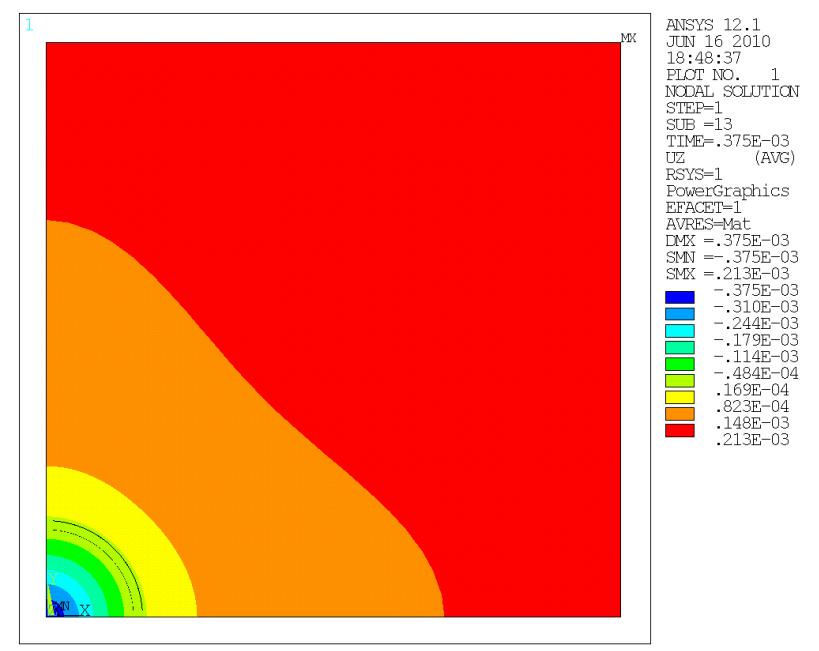

Figure 9. Displacements of model 1 
The finite stiffness of the test machine leads to additional displacements, which have been determined by means of a calibration process. The resulting correction curve has been added to the numerical results before comparing experimental and numerical results. The FEM has been developed using one sample tested with a thickness of $210.59 \mu \mathrm{m}$. The comparison of test results and numerical results (figure 10) shows the good adjustment obtained with this model.

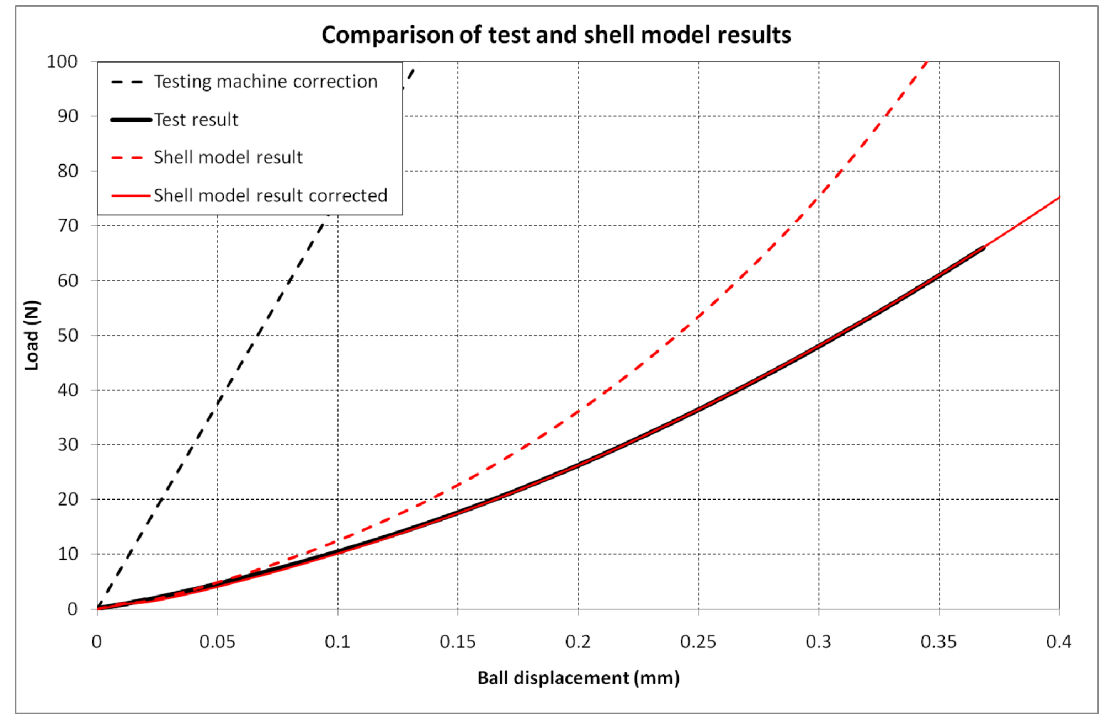

Figure 10. Comparison of test and FEM results

The main disadvantage of this model is that the analysis of the stress variation through the thickness is very limited. In figure 11 the stress state for a displacement of $0.375 \mathrm{~mm}$ is shown near the contact zone with the ball for both top and bottom side.

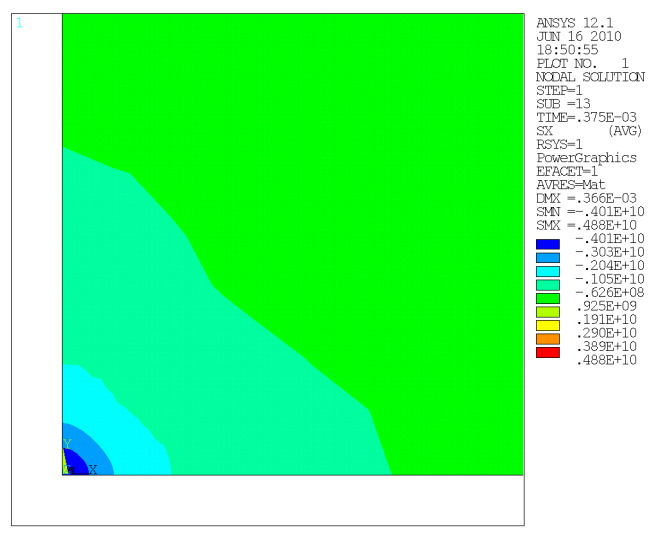

Top side

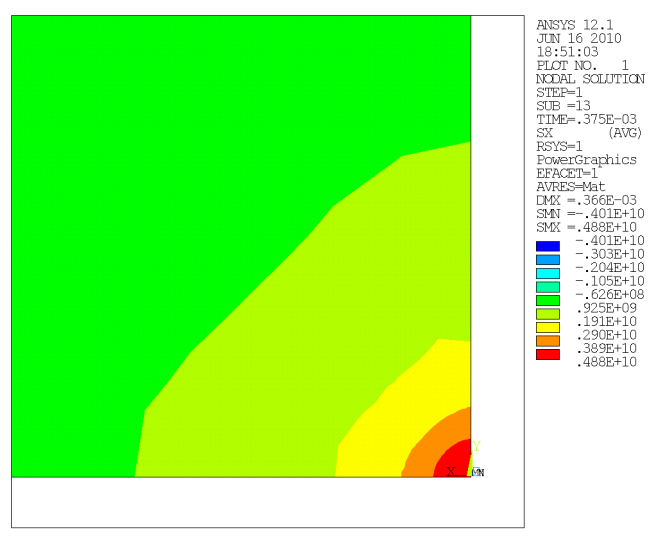

Bottom side

Figure 11. Zoom of the stress state near the contact zones 
Studying the stress evolution through two different directions of the wafer, it can be concluded that stresses near the corners and edges can be neglected (figure 12).

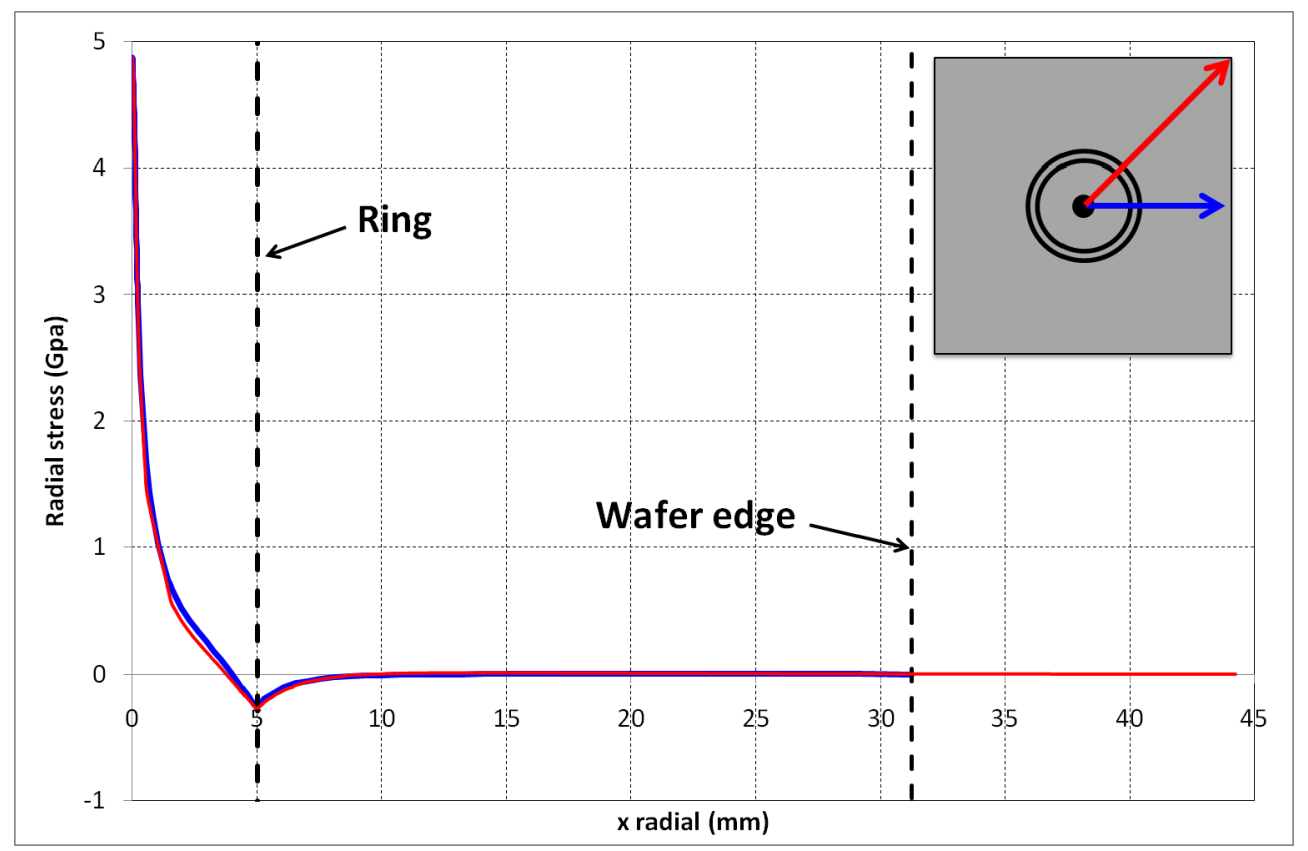

Figure 12. Comparison of stresses in two different directions

In light of these results, an axisymmetric model has been developed to simulate the ball on ring test.

\subsection{Model 2: Axisymmetric}

The second model presented is an axisymmetric model. It presents some advantages as can be the reduced calculation time and the possibility to evaluate the stress state through the thickness of the wafer in more detail. Moreover, the results shown in figure 12 support the development of this model since stresses near the corners can be neglected. On the other hand, this model assumes some hypotheses that are not really true as can be the round shape of the wafer or an isotropic behaviour. To build this model, a value of $165.6 \mathrm{GPa}$ has been taken for the elastic constant $\mathrm{E}$ and the Poisson coefficient has a value of 0.23 . The assumption of an isotropic behaviour using the highest value of the elastic tensor is quite usual in the analysis of multicrystalline silicon wafers ([11]) where the different orientations of the crystals at least partly eliminate the anisotropic behaviour.

Eight node plane elements have been employed. The mesh size is very small because the number of elements (figure 13) and the calculation time is not a problem in this model. There are eight layers of elements in order to analyze the stress evolution through the thickness. This model has 5909 elements and 15457 nodes. The calculation has taken 114 seconds to be completed. 


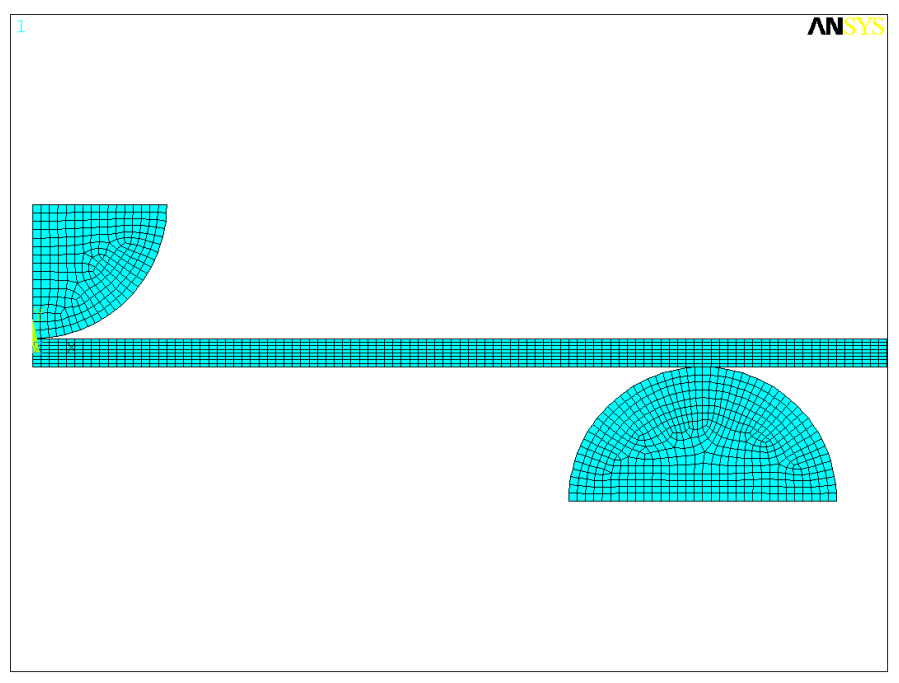

Figure 13. Mesh of the model 2

The adjustment to the test results is not as good as the shell elements model's one (figure 14).

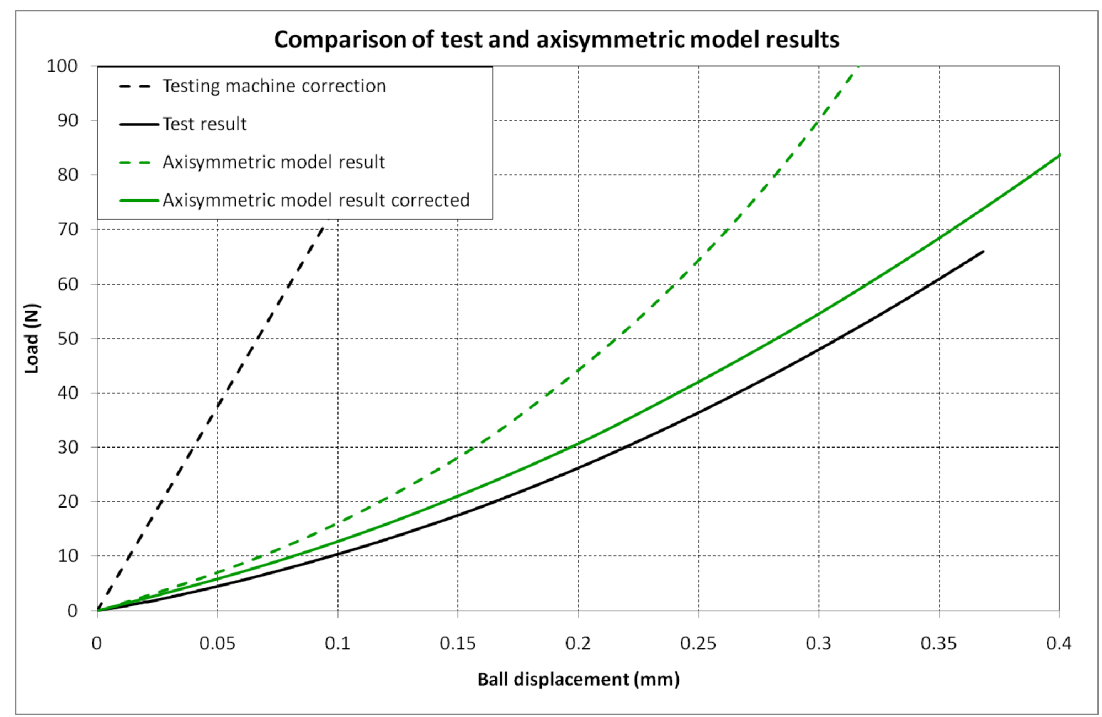

Figure 14. Comparison of test and FEM results

This model presents obviously a more rigid behaviour than the previous one and the test result. This is due to the use of the highest value of the elastic tensor as Young's modulus. Even so, the big advantage of this model is the reduced calculation time and the detailed information about the evolution of the stresses through the thickness. In figure 15 the stress distribution across the thickness is shown and in figure 16 a comparison of the stress information just below the ball between model 1 and 2 is presented. 


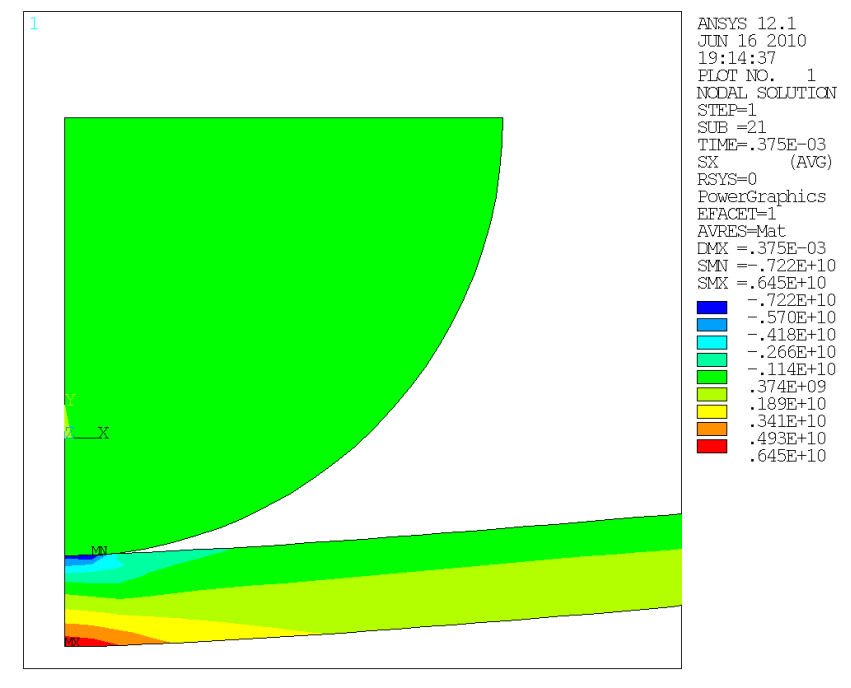

Figure 15. Stresses through the thickness in the axisymmetric model

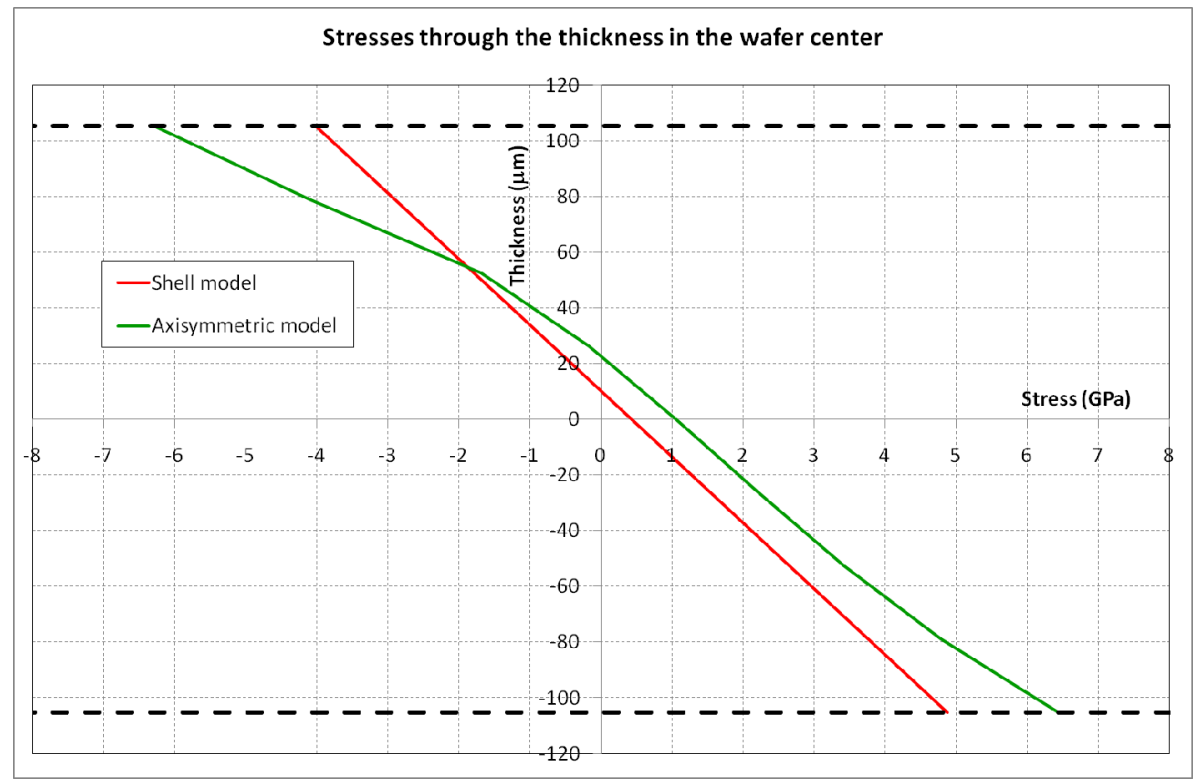

Figure 16. Comparison of the stress information between the two models

To summarize, the axisymmetric model presents some disadvantages simulating the behaviour of the wafer but at the same time, it gives more detailed information on the stress distribution across the thickness.

\subsection{Model 3: 3d solid model}

In order to get a fuller view of the test and to evaluate the advantages of each of the simplified models presented before, a solid model has been developed. This model permits to obtain information of the stresses through the thickness taking into account the anisotropic behaviour and the real shape of the wafer. 
Solid elements with twenty nodes have been employed. As in the shell model case, the mesh size varies depending on the zone of the wafer (figure 17).

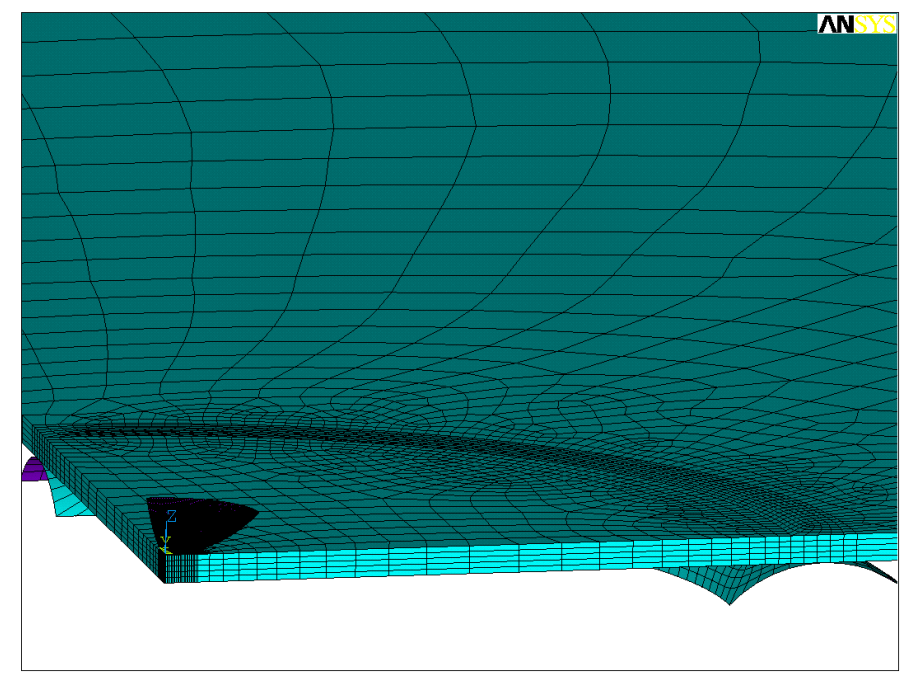

Figure 17. Mesh of the solid model

The main disadvantage of this model is the high calculation time. Because of it, only five layers of element have been employed in this analysis. This model has 34195 elements and 105627 nodes. It takes 51396 seconds to complete the simulation. The result fits quite well to the test result (figure 18). In this case, the adjustment of the previous cases has been kept in order to compare with the fitting got with the solid model.

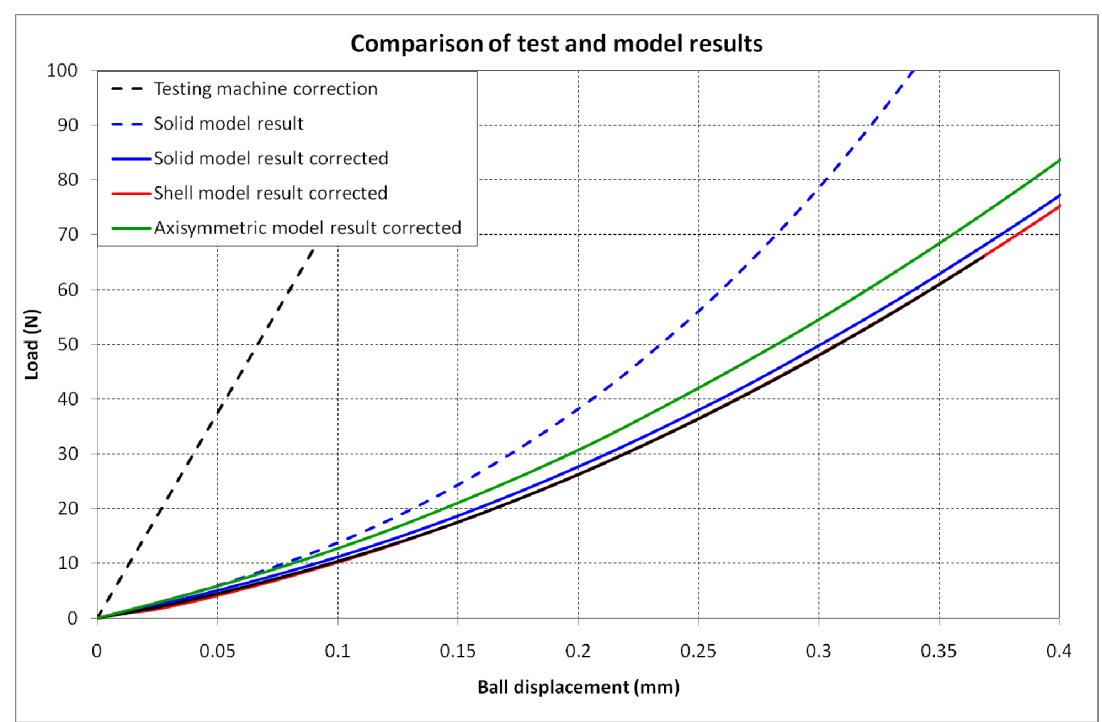

Figure 18. Comparison of test and numerical results 
Moreover, the solid model permits to determine the stress distribution through the thickness. In figure 19 stresses below the ball are shown both in top and bottom side.
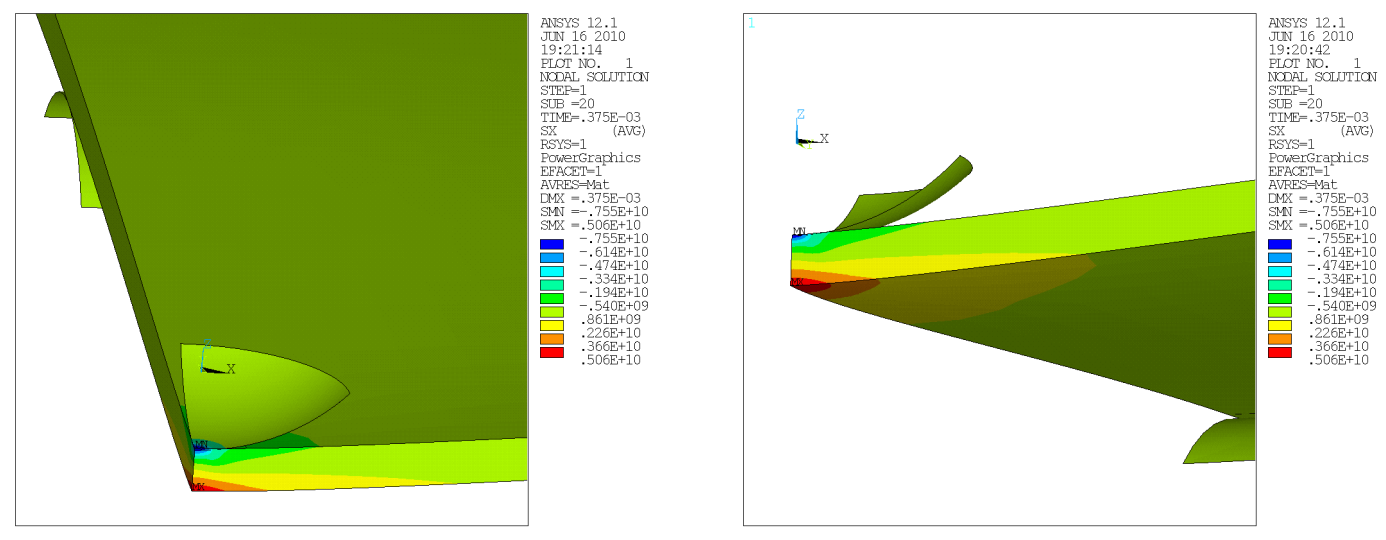

Figure 19. Stresses in the wafer below the ball

In figure 20, the evaluation of the stress through the thickness in the centre of the wafer is presented for all models. It can be seen that the shell model is well suited to determine the maximum tensile stress. In order to calculate the compression stress more element layers seem to be necessary.

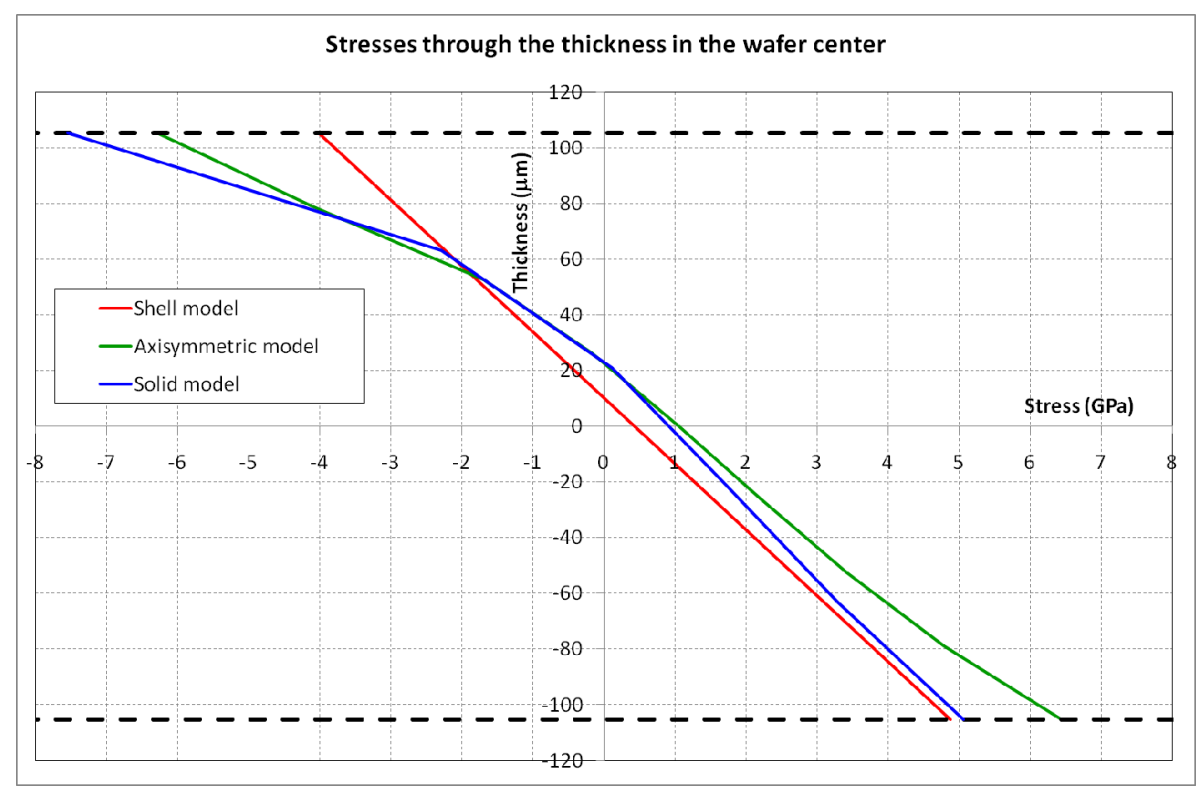

Figure 20. Stress through the thickness

Therefore, the solid model gives the most realistic and accurate results but it takes much more time than the simplified ones. Moreover, it requires much more memory than the previous ones. 


\section{Error estimation depending on the model}

In order to get an estimation of the error due to the numerical model chosen to analyze the test results, the whole study for a set of wafers has been carried out.

Once a model is developed, the next step is to get the fracture stress of all tests. In this study, for the analytical method the fracture stress has been calculated for each test. However, for the FE models only one of each different model has been developed. Since the thickness of all samples is very similar, the fracture stress of each test has been obtained calculating the elastic energy of the sample before fracture and obtaining the stress in the numerical model corresponding to this elastic energy.

When all stress fractures are obtained, a statistical study is carried out ([6], [7], [9]). For brittle materials the Weibull distribution is commonly used to evaluate statistically the results. The probability of failure is defined as ([3]):

$$
P_{f}=1-e^{-\left(\sigma / \sigma_{\theta}\right)^{m}}
$$

The parameter $\sigma_{\theta}$ represents the characteristic fracture stress at which $63.2 \%$ of all samples fail. The Weibull module $\mathrm{m}$ informs about how scattered the results are.

The Weibull fitting of the fracture stresses of all tests obtained with different methods is represented in figure 21 .

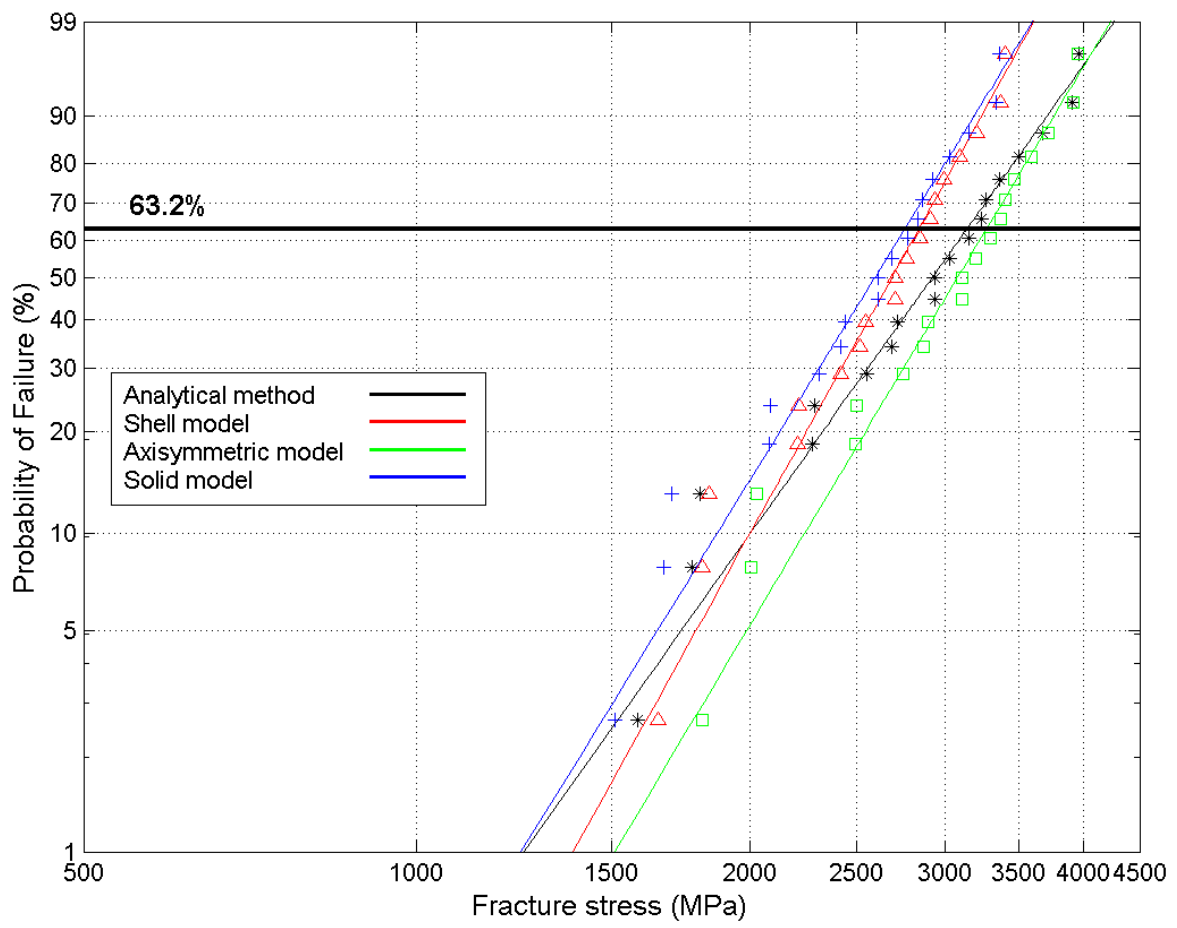

Figure 21. Adjustment to Weibull distributions 
The way chosen to analyze the test results has an important influence on the fracture stress obtained. As could be imagined from figure 20, fracture stress obtained through solid model and shell model are really close. However, axisymmetric model presents higher values of fractures stress due to the more rigid behaviour imposed in the model. Analytical method results are more scattered than the other ones. This may be explained since for analytical method the fracture stress has been calculated from the expression (1) for each test while for numerical models one unique model has been developed and the fracture stress has been calculated from an interpolation of the elastic energy. The thickness in the numerical models hasn't been taken into account.

\section{Summary and conclusions}

The ball on ring test is widely used in mechanical characterization of silicon wafers. In this paper, different ways to simulate this test are presented. First of all, it's important to take the anisotropic behaviour of monocrystalline silicon wafers into account. Moreover, the test is detailed and results for a set of samples are shown. The non-linear behaviour of the samples during the test can be seen clearly. This is due to the large displacements and the contact between the sample, the ball and the ring.

The first method to analyze the test results consists in using analytical expressions taken from the literature. This method is inappropriate since the anisotropic behaviour and the non-linearities present in the test aren't taken into account. However, it's a quick and easy way to analyze test results and can be useful to make comparisons between different sets of wafers.

More realistic models need to consider the non-linearities. Therefore, the Finite Element Method has been employed for the simulation of the tests.

The first model developed is a 3-d model with shell elements. The calculation is quite fast and results fit really well to the test. It's found that the stress gradient is very high near the contact zone between the sample and the ball. The information about stress distribution across the thickness of the wafer in this zone is very limited in this model. It has been shown that the stress distribution near the corner can be neglected so an axisymmetric model can be developed.

The axisymmetric model permits to get much more information about the stress distribution in the wafer centre than the previous one. The anisotropy cannot be included in this model and circular shape of the sample has to be assumed. For the isotropic behaviour, the highest value of the elastic tensor is taken as Young's modulus. Therefore, the model behaves more rigid than the previous one. However, the stress distribution can be studied and it's shown that the compression in the top side is really important.

In order to establish reference values, a 3-d model using solid elements has been developed. This model takes into account all the features present in the problem. However, it takes a lot of time to complete the calculation and it requires a lot of memory. Results from this model fit quite well to test results and the stress distribution across the thickness is very similar to the one obtained with the shell 
model at the bottom and more similar to the one obtained with the axisymmetric model at the top.

In order to get an estimation of the error that can be expected in terms of characteristic fracture stress depending on the model chosen for the analysis, a Weibull fit has been made. As only one thickness has been considered in all models, the fracture stress for each test has been calculated by means of an interpolation of the elastic energy at the moment of fracture. Finally, fracture stresses calculated from different ways of the whole set of samples have been adjusted to a Weibull distribution.

Table I shows a comparison of all methods employed to simulate the ball on ring test.

\begin{tabular}{l|ccccc}
\hline Model/Method & $\begin{array}{c}\text { Number of } \\
\text { elements }\end{array}$ & $\begin{array}{c}\text { Number } \\
\text { of nodes }\end{array}$ & $\begin{array}{c}\text { Calculation } \\
\text { time }(\mathrm{s})\end{array}$ & $\begin{array}{c}\text { Characteristic Fracture } \\
\text { stress }\left(\sigma_{\theta} \mathrm{MPa}\right)\end{array}$ & $\begin{array}{c}\mathrm{m} \\
\text { module }\end{array}$ \\
\hline Analytical & - & - & - & 3142.02 & 4.98 \\
Shell & 20239 & 11530 & 440 & 2842.36 & 6.39 \\
Axisymmetric & 5909 & 15457 & 114 & 3272.79 & 5.94 \\
Solid & 34195 & 105627 & 51396 & 2760.75 & 5.76 \\
\hline
\end{tabular}

Table I. Comparison of all methods and models

These results show that the shell model is well suited to simulate the tests because the results are close to the ones obtained with the solid model and the calculation time and memory requirements are not so high. In order to develop one numerical model for each test, the shell model should be employed. On the other hand, one solid model would be helpful in order to get a better understanding of the behaviour of the sample during the test. To develop one solid model for each test is impractical due to the high calculation time.

Analytical methods appear inappropriate since they don't reflect the particularities of the material and the test. They could be useful for a quick comparison of different sets of wafers.

The axisymmetric model gives the highest results due to the value chosen for the Young's modulus. It could be a reliable alternative for the analysis of multicrystalline silicon wafers since they're considered as isotropic. In the case of monocrystalline silicon wafers, the anisotropic behaviour has a quite important impact on the final results.

\section{Acknowledgement}

This work has been made under the sponsorship of the University State Secretary, belonging to Spanish Ministry of Science and Innovation, in its contract TEC200806798-C03-03. The presented results have been obtained in cooperation with Instituto de Energía Solar (IES) and Teknologia Mikroelektronikoaren Institua (TIM). 


\section{References}

[1] A. Luque, S. Hegedus, "Handbook of Photovoltaic Science and Engineering", John Wiley \& Sons Ltd, West Sussex, England, 2003.

[2] J. C. Jimeno, V. Rodríguez, R. Gutiérrez, F. Recart, G. Bueno, F. Hernando, "Very Low Thickness Monocrystalline Silicon Solar Cells", Sixteenth European Photovoltaic Solar Energy Conference, Glasgow, Scotland, 2000.

[3] W. Weibull, "A Statistical Theory of the Strength of Materials", Proceedings of the Royal Swedish Institute of Engineering Research Nr. 151, 1939

[4] J. Barredo, L. Hermanns, A. Fraile, J. C. Jimeno, E. Alarcón. "The influence of different surface treatments on the mechanical strength of silicon wafers". 22nd European Photovoltaic Solar Energy Conference, Milan, Italy, 2007

[5] D. Broek, "Elementary Engineering Fracture Mechanics", Martinus Nijhoff Publishers, 1986

[6] J. Barredo, A. Fraile, E. Alarcón, "Study of the Brittle Fracture of Monocrystalline Silicon Wafers", in B.H.V. Topping, (Editor), "Proceedings of the Tenth International Conference on Civil, Structural and Environmental Engineering Computing", Civil-Comp Press, Stirlingshire, UK, Paper 104, 2005. doi:10.4203/ccp.81.104

[7] B. Cotterell, Z. Chen, J. B. Han, N. X. Tan, "The Strength of the Silicon Die in Flip-Chip Assemblies", Journal of electronic packaging, ASME, USA, 2003.

[8] D. K. Shetty, A. R. Rosenfield, P. McGuire, G. K. Bansal, W. H. Duckworth. "Biaxial Flexure Test for Ceramics", Ceram. Bull., vol. 59, no. 12, 1980 (1193-1197).

[9] S. Schoenfelder, A. Bohne, J. Bagdahn. "Comparison of test methods for strength characterization of thin solar wafer". 22nd European Photovoltaic Solar Energy Conference, Milan, Italy, 2007

[10] A. Börger, P. Supancic, R. Danzer. "The ball on three balls test for strength testing of brittle discs: stress distribution in the disc". Journal of the European Ceramic Society 22, 2002 (1425-1436).

[11] S. Dumoulin, S. Gouttebroze, M. M'Hamdi. "Modelling of intergranular stresses during elastic deformation of multi-crystalline silicon" 23rd European Photovoltaic Solar Energy Conference, Valencia, Spain, 2008

[12] D. Munz, T. Fett. "Ceramics. Mechanical Properties, Failure Behaviour, Materials Selection”, Springer Series in Materials Science, 1999 